\title{
IMPUESTOS AMBIENTALES SOBRE LOS DESECHOS SÓLIDOS EN TABASCO: EN PRO DEL MEDIO AMBIENTE
}

Environmental Taxes on Solid Waste In Tabasco: For The environment

\author{
Germán Martínez Prats*, Carlos Mauricio Tosca Vidal** y Aldo Alexis \\ Juárez Domínguez ${ }^{* * *}$
}

\section{RESUMEN}

En México existe la Ley General de Cambio Climático (LGCC), la cual plantea estrategias para disminuir a largo plazo los efectos del cambio climático mediante reducción de las emisiones de gases que dañan el medio ambiente y la implementación en la sociedad de la cultura de adaptación de los impactos que deriven del cambio climático, aplicando en el caso de incumplimiento de sus disposiciones las multas y sanciones pertinentes (Cámara de Diputados del H. Congreso de la Unión, 2018b). Sin embargo, la LGCC solo maneja intervalos de los cuales se establecen las multas e infracciones que deben exigir las autoridades mediante sus facultades, sin asignar el método de aplicación, salvo por los jueces calificadores asignados. El objetivo de esta investigación fue analizar si existen los medios para generar impuestos ambientales sobre los desechos sólidos. Esta investigación tiene un alcance descriptivo, debido a que se describe el fenómeno con las teorías e investigaciones válidas que se obtuvieron. La investigación fue de tipo documental, basando en la recolección de datos en fuentes secundarias, en libros y bases de datos.

Palabras Clave: Impuesto, Cambio climático, Basura, Desechos sólidos, Medio ambiente.

RECIBIDO: Noviembre 2018

ACEPTADO: Marzo 2019

\footnotetext{
* Dr. En Gobierno y Administración Pública Universidad Juárez Autónoma de Tabasco Villahermosa, Tabasco, México. Correo electrónico: germanmtzprats@ hotmail.com

** Mtro. en Contaduría. Universidad Juárez Autónoma de Tabasco. Villahermosa, Tabasco, México. Correo electrónico: toscavidal@ hotmail.com

${ }_{* * *}$ Mtro. en Derecho Fiscal. Universidad Juárez Autónoma de Tabasco. Villahermosa, Tabasco, México. Correo electrónico: Aldojuarezd@hotmail.com
} 
Impuestos ambientales sobre los desechos sólidos en tabasco: en pro del medio ambiente

\begin{abstract}
In Mexico there is the General Law on Climate Change (LGCC), which proposes strategies to reduce the effects of climate change in the long term by reducing emissions of gases that harm the environment and the implementation in society of the culture of adaptation of the impacts that derive from climate change, applying in the case of noncompliance with its provisions, the fines and pertinent sanctions (Chamber of Deputies of the H. Congress of the Union, 2018b). However, the LGCC only handles intervals of which the fines and infractions are established that the authorities must demand through their faculties, without assigning the method of application, except for the assigned qualifying judges. The objective of this research is to analyze if there are means to generate environmental taxes on solid waste. This research has a descriptive scope, because the phenomenon is described with the theories and valid investigations that were obtained. The research was documentary, based on data collection in secondary sources, in books and databases.
\end{abstract}

Key Words: Tax, Climate change, Garbage, Solid waste, Environment.

\title{
Introducción.
}

A nivel internacional, los países desarrollados mantienen en su legislación fiscal impuestos regulatorios para la contaminación de sus habitantes, y una aplicación estricta de las multas y sanciones correspondientes a estos, teniendo como ejemplo principal a los países vecinos de Estados Unidos y Canadá (Borrego, 2006).

Sin embargo, en México debido a diversos factores, la falta de aplicación de las sanciones contenidas en la Ley General de Cambio Climático(LGCC), y una escueta difusión e implementación de una cultura ambiental positiva en los distintos estratos de la sociedad, aun se tiene un índice de alto de deterioro ambiental (Borrego, 2006).

Con paso del tiempo, los daños al medio ambiente van en aumento, ocasionado por la falta de acciones y culturas ecológica, a la extracción de recursos naturales de forma excesiva y a los altos índices de contaminación.

De acuerdo Instituto Nacional de Estadística y Geografía [INEGI] el cálculo del Producto Interno Neto Ajustado Ambientalmente permite conocer el costo económico que se asumirán por los daños ambientales. En 2015 éste fue equivalente al 5\% del PIB a precios de mercado. Dicho rubro se refiere a la suma de los costos por el agotamiento de los recursos naturales y la degradación ambiental, que alcanzó un 
Germán Martínez Prats, Carlos Tosca Vidal y Aldo Juárez Domínguez.

Telos Vol. 21, No. 2 (2019). 333-346

monto de 907,473 millones de pesos (Centro de Estudios de las Finanzas Públicas, 2016).

En Tabasco, existe la Ley de Protección Ambiental del Estado de Tabasco (LPAET), en su artículo 301 de las sanciones administrativas que pueden aplicar las autoridades competentes en materia ambiental, donde se establecen las multa que van desde veinte hasta cincuenta mil unidades de medidas y actualización (UMA), que anteriormente se utilizaba como referencia los Salarios Mínimo General (Cámara de Diputados del H. Congreso del Estado de Tabasco, 2017).

El objetivo del presente trabajo es analizar si en Tabasco existen los medios para la generación impuestos ambientales sobre los desechos sólidos, de acuerdo a la legislación actual y la aplicación de los impuestos verdes en el mundo. Para ello en el apartado de desarrollo se analizará el fundamento de los impuestos en la carta Magna, adicionalmente se aborda la relación entre los impuestos y el medio ambiente así como la historia de los impuestos verdes en el mundo. Posteriormente se analizará el impacto del cambio climático y su relación con los desechos sólidos. Finalmente, se describe la metodología empleada para la investigación, y las conclusiones a través del análisis de los aspectos mencionados anteriormente.

\section{Desarrollo.}

Gaudumet y Molinier (1996), mencionan que las finanzas públicas constituyen una rama del derecho público que tiene por objeto el estudio de las reglas y las operaciones relativas a los fondos públicos (citado en Soto, 2007).

Debido a los grandes cambios, los sistemas fiscales modernos se han definido en la determinación personal de los impuestos por parte de los contribuyentes. Uno de los fundamentos de esta práctica es la presunción de que los contribuyentes conoce toda su información fiscal relevante y la cultura fiscal de cooperación con las autoridades recaudatorias dentro de un marco no coercitivo de administración fiscal (Ruiz, 2016). 
Impuestos ambientales sobre los desechos sólidos en tabasco: en pro del medio ambiente

Los mecanismos mediante los cuales el estado obtiene sus recursos, están establecidos en la ley de ingresos de la federación, siendo algunos de estos la explotación de sus propios bienes, el manejo de sus empresas públicas; la recaudación, fiscalización y gestión de las contribuciones normadas en las leyes y reglamentos emanados por este, y por último la solicitud y administración de créditos internos y externos (Delgadillo, 2005).

La obligación de contribuir al gasto público se fundamenta en el artículo 31 fracción IV de la Constitución Política de los Estados Unidos Mexicanos (CPEUM), el cual menciona: "Son obligaciones de los mexicanos a contribuir para los gastos públicos, así de la Federación, como de los Estados, de la Ciudad de México y del Municipio en que residan, de la manera proporcional y equitativa que dispongan las leyes" (Cámara de Diputados del H. Congreso de la Unión, 2018a, p.41).

Relativos a las competencias tributarias, la CPEUM establece en el artículo 73, fracción VII que la entidad tiene competencia para imponer contribuciones necesarias para cubrir el presupuesto. Derivado de lo anterior, las fuentes impositivas que solo la federación puede gravar, contenidas en la fracción XXIX del artículo en mención (Cámara de Diputados del H. Congreso de la Unión, 2018a). Sin embargo, puede gravar otras fuentes si es necesario para generar los ingresos para cubrir los gastos públicos en los términos de la fracción VII del artículo 73 constitucional (Cámara de Diputados del H. Congreso de la Unión, 2018a). Es decir, se menciona que la parte tributaria no tiene límites de acuerdo a las necesidades de presupuesto del país señaladas en la fracción XXIX si estás no son atendidas de manera oportuna, sin limitar a generar otros impuestos, en la fracción VII lo faculta para decretar todas las contribuciones, con el fin de cubrir el gasto público, como se detalla en las fracciones del artículo 73 descritas en líneas anteriores (Cámara de Diputados del H. Congreso de la Unión, 2018a). Por lo tanto, y expuesto lo anterior, el sistema jurídico mexicano y el origen de la obligación que tienen todos los mexicanos para contribuir al gasto público permite entonces que las autoridades de la administración pública puedan generar las normativas pertinentes, 
Germán Martínez Prats, Carlos Tosca Vidal y Aldo Juárez Domínguez.

Telos Vol. 21, No. 2 (2019). 333-346

así como acciones congruentes establecidas en las políticas públicas desarrolladas para cada institución.

\section{Los impuestos.}

Los impuestos forman parte de los ingresos públicos, tan solo en la ley de ingresos de la federación para el ejercicio del 2018 se completa que los ingresos por este concepto son el $82.49 \%$, siendo la parte más sustancial de los ingresos que cuenta el estado para cubrir las necesidades de la sociedad (Cámara de Diputados del H. Congreso de la Unión, 2017).

Por lo tanto, los ingresos públicos hacen alusión a las percepciones que el Estado recibe, sean en efectivo o en especie, y la contribución, que forman parte de lo que integra los ingresos públicos que además incluye aportaciones de particulares, pagos por el uso de servicios públicos, multas, entre otros (Rosas y Santillán, 1962; Arrioja, 1997).

El Código Fiscal de la Federación en el artículo $2^{\circ}$ expresa que “Impuestos son las contribuciones establecidas en ley que deben pagar las personas físicas y morales que se encuentran en la situación jurídica o de hecho prevista por la misma y que sean distintas de las señaladas en las fracciones II, III y IV (Cámara de Diputados del H. Congreso del Estado de Tabasco, 2017, p. 5).

\section{El medio ambiente y los impuestos.}

El medio ambiente es un "Sistema global complejo, de múltiples y variadas interacciones, dinámico y evolutivo, formado por los sistemas físico, biológico, social, económico, político y cultural en que vive el hombre y demás organismos" (Labandeira, León, y Vázquez, 2007, p. 4).

La sobreexplotación del mundo contemporáneo en el ambiente , ha generado la ruptura del equilibrio ecologico (Santiago, 2009). En Mexico, la cobertura de los 
Impuestos ambientales sobre los desechos sólidos en tabasco: en pro del medio ambiente

ecosistemas del pais se redujo al 62\% en 1976, 1993 el 54\% y 38\% para el 2002 donde la mayoria fue en las zonas tropicales (Comisión Nacional para el Conocimiento y Uso de la Biodiversidad, 2009).

Para revertir esta situación, los gobiernos han implementado impuestos para el mantenimiento y preservación del ambiente conocidos como impuestos verdes. De acuerdo a varios autores también se conocen como impuestos ambientales, eco tributos, e impuestos con incidencia ambiental (Pitrone, 2014).

\section{El origen del impuesto ambiental}

Una de las primeras conceptualizaciones de impuesto ambiental es de la Organización para la cooperación y el desarrollo Económicos (OCDE) en la Implementation Strategies for Environmental Taxes, en donde eran creados para cumplir un objetivo ambiental específico y eran denominados explícitamente como impuestos ambientales, o impuestos creados bajo ninguna razón vinculada al medio ambiente, pero que tenían un impacto en el mismo (Organización para la Cooperación y el Desarrollo Económico [OCDE], 2001).

Estos impuestos han servido como motor del cambio con otras políticas para que países como Holanda o Suiza adopten una mejor cultura en cuanto a cómo se desarrollan y a su vez, mejorar la calidad de vida de sus habitantes (OCDE, 2001). En el presente, la fiscalidad ambiental se ha convertido en un elemento fundamental dentro de las políticas ambientales en los países de la OCDE (Jofra y Puig-Ventosa, 2014).

En la figura 1, se refleja que los impuestos ambientales están dirigidos a la energía con el $71 \%$ del resto de los impuestos ambientales. Esto se debe al hecho de que fueron los primeros impuestos que los países de la OCDE introdujeron en sus sistemas fiscales y al gran potencial recaudatorio que tienen estos con respecto a otros impuestos ambientales (Varela y García 2010).

Seguido de los impuestos ambientales a la energía, son los impuestos sobre el transporte, que, a pesar de no tener la importancia de los impuestos sobre la energía, 
representan casi el 25\% del total de los impuestos ambientales Y, por último, los impuestos sobre la contaminación y los recursos, que su presencia comparada con los otros dos grupos es pequeña (Varela y García, 2010).

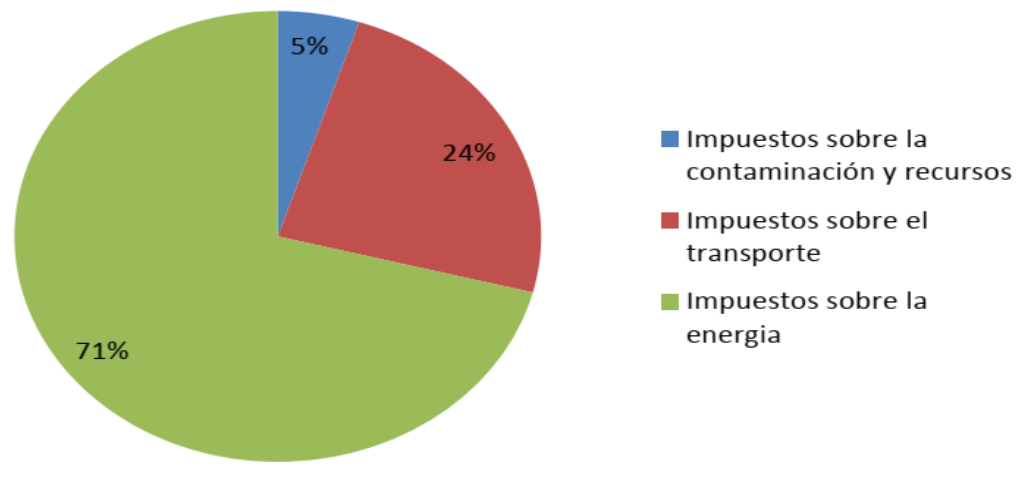

Figura 1. Tipo de impuestos ambientales en los países de OCDE.

Fuente: Elaboración propia a partir de datos de Varela y García 2010.

En México, estos conceptos son relativamente nuevos, como se muestra en la figura 2, en 2012 fue el último lugar en recaudación de este tipo de impuestos en la OCDE, y la participación en la misma ayudó a crear una Ley que da la pauta para la creación de los impuestos ambientales. La LGCC, que tiene como principales objetivos garantizar el derecho a un medio ambiente y regular las emisiones para lograr la estabilización de sus concentraciones en la atmósfera, para lograr alcanzar un nivel que nos impida inferencias peligrosas en el sistema climático. Su primera meta es que para el año 2020 al menos se reduzca un 30\% de las emisiones en comparación con el año 2000 y para el 2050, su meta será el 50\% de reducción (Cámara de Diputados del H. Congreso de la Unión, 2018b). 
Impuestos ambientales sobre los desechos sólidos en tabasco: en pro del medio ambiente

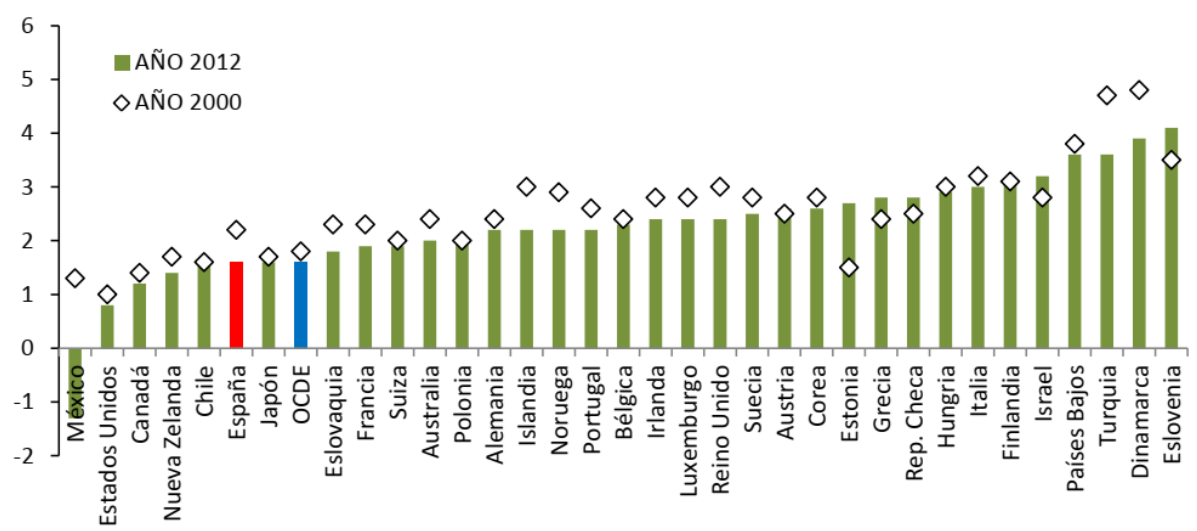

Figura 2. Nivel de recaudación de impuestos ambientales de los países de la OCDE. Fuente: Elaboración propia a partir de datos de la OCDE.

La LGCC promueve el cumplimiento de la legislación, y a la vez, elabora los instrumentos que se replican en los estados, en los cuales se establecen las funciones y autoridades que aplican los preceptos legales y estatales en materia de promoción del cuidado y del medio ambiente. En el artículo $7^{\circ}$ sobre las atribuciones de la federación, en su fracción XX menciona que la Federación diseñara y promoverá ante las dependencias y entidades competentes, las políticas públicas e instrumentos económicos, fiscales, financieros y de mercado en materia del cuidado del medio ambiente (Cámara de Diputados del H. Congreso de la Unión, 2018b).

\section{Cambio climático y producción de basura}

La basura es unos de los principales causales del cambio climático debido a la contaminación de suelo, aire y agua, tan solo en Tabasco en el año 2017 de acuerdo Censo Nacional de Gobiernos Municipales y Delegacionales publicado por el INEGI (2018), se generan aproximadamente 1,990,960 kilogramos en promedio diarios, equivalentes a 830 gramos por habitante al día.

El cambio climático es un cambio de clima atribuido directa o indirectamente a la actividad humana que altera la composición de la atmosfera mundial y que se suma 
a la variabilidad natural del clima observando durante periodos comparables (Organización de las Naciones Unidas, 1992). El cambio climático también hace referencia a las modificaciones de cualquier aspecto de la tierra, como el agua, la temperatura, tormentas ciclones entre otros (Miller, 2007).

\section{Tabla 1. Impacto del cambio climático en México}

\begin{tabular}{|c|c|}
\hline Sistemas y sectores & Descripción \\
\hline Agricultura & $\begin{array}{l}\text { Más de } 80 \text { por ciento de los suelos agrícolas de México presenta algún } \\
\text { grado de erosión, debido a los monocultivos y la deforestación, } \\
\text { promovida esta última para la ganadería extensiva y la producción de } \\
\text { pasturas. }\end{array}$ \\
\hline Hídrico & $\begin{array}{l}\text { El } 96.98 \text { por ciento de México es muy vulnerable a la desertificación: } \\
48.05 \text { por ciento del territorio es susceptible de ser afectado por } \\
\text { procesos de degradación de tierras en alto grado y } 48.93 \text { por ciento } \\
\text { en grado moderado. }\end{array}$ \\
\hline Bosques & $\begin{array}{l}\text { México ocupa el quinto lugar mundial en deforestación. Cada año } \\
\text { pierde alrededor de } 600 \text { mil hectáreas de bosques y selvas, lo que } \\
\text { equivale a perder cada año una superficie equivalente a cuatro veces } \\
\text { el D.F. La pérdida de bosques y su deterioro contribuyen con } 14 \text { por } \\
\text { ciento de las emisiones nacionales de gases de efecto invernadero } \\
\text { (GEI) del país. }\end{array}$ \\
\hline Manglares & $\begin{array}{l}\text { El manglar está desapareciendo a una tasa promedio de } 2.5 \text { por ciento } \\
\text { anual, lo que equivale a poco más de } 4.43 \text { hectáreas cada día o a seis } \\
\text { campos de fútbol al día. El Instituto Nacional de Ecología y Cambio } \\
\text { Climático indica que si la tasa de pérdida se mantiene constante, para } \\
\text { el año } 2025 \text { habrá desaparecido entre } 40 \text { y } 50 \text { por ciento de la } \\
\text { superficie de manglar estimada para el año } 2000 \text {. }\end{array}$ \\
\hline $\begin{array}{l}\text { Ecosistemas } \\
\text { marinos }\end{array}$ & $\begin{array}{l}\text { En } 1950 \text { había } 44 \text { especies de mamíferos marinos en México. Hoy } \\
\text { todas están en alguna categoría de riesgo y dos se consideran extintas. } \\
\text { De la vaquita marina, único mamífero marino endémico de México, } \\
\text { quedan menos de } 150 \text { ejemplares. Incluso una especie recién } \\
\text { descubierta en el Golfo de California, el tiburón Mustelus hacat, ya } \\
\text { se encuentra amenazada. }\end{array}$ \\
\hline Transporte & $\begin{array}{l}\text { El sector transporte emite } 18 \text { por ciento de los GEI en México. En la } \\
\text { Ciudad de México esta proporción llega al } 43 \text { por ciento, y los } \\
\text { principales contribuyentes son los autos particulares. La norma que } \\
\text { regula la concentración máxima de ozono en el aire es rebasada } 233 \\
\text { días al año en la zona metropolitana del valle de México, } 66 \text { días en } \\
\text { Guadalajara, } 32 \text { días en Monterrey y } 22 \text { días en Toluca. }\end{array}$ \\
\hline
\end{tabular}


Impuestos ambientales sobre los desechos sólidos en tabasco: en pro del medio ambiente

\section{Tabla 1. Impacto del cambio climático en México}

\begin{tabular}{|c|c|}
\hline Sistemas y sectores & Descripción \\
\hline Agua & $\begin{array}{l}\text { México tiene actualmente } 653 \text { acuíferos, de los cuales, } 288 \text { están } \\
\text { disponibles, lo que representa sólo el } 44 \text { por ciento de ellos. Pese a } \\
\text { que aumentó el número de acuíferos disponibles (sólo había } 188 \text { en } \\
\text { el 2003), también creció dramáticamente el número de los que están } \\
\text { siendo sobreexplotados: hace } 5 \text { años había } 65 \text { zonas de agua } \\
\text { subterránea sobreexplotadas y en la actualidad, son } 101 \text { las zonas } \\
\text { sujetas a una extracción superior a la recarga que reciben. De estas } \\
\text { cuencas se extrae el } 58 \text { por ciento del agua subterránea para todos los } \\
\text { usos del país. El monitoreo de la Comisión Nacional del Agua } \\
\text { (CONAGUA) han arrojado los siguientes resultados: en el rubro } \\
\text { DBO5, la CONAGUA admite que } 14 \text { por ciento del agua está } \\
\text { contaminada o fuertemente contaminada, mientras que para el DQO, } \\
\text { se reconoce un de } 32.5 \text { por ciento de nivel de contaminación. Para los } \\
\text { SST, la dependencia reconoce un } 8 \text { por ciento. }\end{array}$ \\
\hline Fuentes renovables & $\begin{array}{l}\text { El potencial eólico aprovechable en México varía entre } 5 \text { mil y } 50 \text { mil } \\
\text { MW. Una evaluación realizada con imágenes satelitales en el Istmo } \\
\text { de Tehuantepec arrojó un potencial de cerca de } 10,000 \mathrm{MW} \text {, } \\
\text { incluyendo posibles instalaciones en el mar. Este potencial no se ha } \\
\text { explotado debido a la falta de claridad en la regulación y a la } \\
\text { incapacidad del Estado para manejar los conflictos sociales de forma } \\
\text { que las inversiones generen beneficio a las comunidades en donde se } \\
\text { instalan los proyectos. }\end{array}$ \\
\hline Pesca & $\begin{array}{l}\text { La pesca de camarón arrastra cada temporada casi } 550,000 \mathrm{~km} 2 \text { en el } \\
\text { Pacífico y } 187,031 \mathrm{~km} 2 \text { en el Golfo de México, lo que equivale a } \\
\text { barrer con todos los animales que habitan en el fondo de la cuarta } \\
\text { parte del mar territorial mexicano. }\end{array}$ \\
\hline Industria Sucia & $\begin{array}{l}\text { Debido a procesos industriales sucios y a la aplicación laxa de las } \\
\text { leyes, hoy tenemos un país saturado de residuos peligrosos. Existe } \\
\text { una relación directa entre el riesgo de efectos adversos a la salud y la } \\
\text { proximidad de la población a los sitios donde se producen o se } \\
\text { encuentran almacenados los residuos peligrosos. A pesar de que está } \\
\text { vigente una Ley General para la Prevención y Gestión Integral de } \\
\text { Residuos, las autoridades mexicanas no han hecho esfuerzo suficiente } \\
\text { para reducir la producción de residuos peligrosos ni para darles un } \\
\text { tratamiento adecuado. Desde el } 2004 \text { y hasta el } 2007 \text {, se generaron en } \\
\text { el país } 8 \text { millones } 826 \text { mil } 546 \text { toneladas de residuos peligrosos. } \\
\text { Apenas en el año } 2000 \text {, la cifra de los residuos peligrosos generados } \\
\text { en el país era mucho menor: } 3 \text { millones, } 705 \text { mil } 846 \text { toneladas de } \\
\text { residuos. }\end{array}$ \\
\hline Calidad del aire & $\begin{array}{l}\text { Por lo menos seis zonas metropolitanas del país (Ciudad de México, } \\
\text { Monterrey, Guadalajara, Toluca, Ciudad Juárez, Puebla y } \\
\text { Salamanca), en las que viven cerca de } 36 \text { millones personas, son }\end{array}$ \\
\hline
\end{tabular}


Germán Martínez Prats, Carlos Tosca Vidal y Aldo Juárez Domínguez.

Telos Vol. 21, No. 2 (2019). 333-346

Tabla 1. Impacto del cambio climático en México

\begin{tabular}{lll}
\hline Sistemas y sectores & Descripción & \\
& $\begin{array}{l}\text { continuamente monitoreadas debido a la contaminación. } \\
\text { Cotidianamente rebasan las normas de calidad del aire. }\end{array}$ \\
\hline Fuente: Adaptado de Greenpeace (2016) con información de Instituto Nacional de Ecología
\end{tabular}
y Cambio Climático.

\section{Metodología.}

La investigación tiene alcance descriptivo, la "investigación que busca especificar propiedades y características importantes de cualquier fenómeno que se analice" (Hernández, Fernandez, y Baptista,2014, p. 92).

La técnica de esta investigación es documental, según Arias (2012, p. 27) esta implica "un proceso basado en la búsqueda, recuperación, análisis, critica e interpretación de datos secundarios". El tipo de investigación es documental monográfica que consiste en el desarrollo amplio y profundo de un tema en específico"(Arias, 2012, p.30) se utiliza la información relevante de las fuentes confiables para obtener la información de la literatura sobre los impuestos verdes en México y en el mundo.

Es de tipo no experimental y transversal debido a que recolecta los datos en momento único. Se busca describir como los impuestos pueden reducir las acciones que generan contaminación en sus diversas formas y de esta manera reducir los efectos del cambio climático.

\section{Conclusiones}

En el mundo los impuestos ambientales son la parte fundamental en la recaudación de la hacienda pública, sin embargo en México existe poca legislación sobre los impuestos verdes, y la mayoría de los impuestos son dirigidos a la energía, como consecuencia del deterioro ambiental se creó la LGCC, que en la fracción XX del artículo 7 (Cámara de Diputados del H. Congreso de la Unión, 2018b) se fundamenta 
Impuestos ambientales sobre los desechos sólidos en tabasco: en pro del medio ambiente

el diseño y aplicación de instrumentos fiscales, para este caso, la creación de impuestos de carácter ambiental.

Con ello y debido al alto índice de desechos sólidos se producen en el Estado de Tabasco y con acciones desiertas para generar una cultura ambiental positiva en los estratos de la sociedad, se considera necesario crear políticas públicas enfocadas a la aplicación estricta de las sanciones contenidas en la Ley de Protección Ambiental del Estado de Tabasco, una mayor metodología en la generación de instrumentos fiscales, como los impuestos ambientales, que permitan mayor recaudación para llevar a cabo las acciones en materia ecológica y medio ambiental de las autoridades competentes, realizar una mayor difusión y promoción de la cultura verde, y de esta manera concientizar del daño al medio ambiente generado por la producción de basura en el estado de Tabasco.

En la presente investigación se analizaron y describieron los mecanismos que permiten generar contribuciones relacionadas con el medio ambiente en el estado de Tabasco, cumpliendo con el objetivo de la investigación que si existen los medios adecuados para la generación de impuestos verdes que coadyuven a la conservación del medio ambiente. Las futuras líneas de investigación que pueden ayudar a la creación de impuestos ambientales es analizar la metodología de aplicación y la creación de programas sociales para la concientización de la conservación del medio ambiente.

\section{Referencias bibliográficas}

Arrioja, Adolfo. (2017). Derecho Fiscal. Themis. México.

Arias, Fidias (2012) El proyecto de Investigación: Introducción a la metodología científica. Episteme. $6^{a}$ edición. Venezuela.

Borrego, Norma. (2006). Las políticas públicas de protección al ambiente en Norteamérica. Revista Mexicana de Estudios Canadienses. Número 11. México, (Pp.163-192.).

Cámara de Diputados del H. Congreso de la Unión. (2017). Ley de Ingresos de la Federación para el ejercicio fiscal de 2018. Diario Oficial de la Federación. México.

Cámara de Diputados del H. Congreso de la Unión. (2018a). Constitución Política de los Estados Unidos Mexicanos. Diario Oficial de la Federación. México. 
Cámara de Diputados del H. Congreso de la Unión. (2018b) Ley General de Cambio Climático. Diario Oficial de la Federación. México.

Cámara de Diputados del H. Congreso del Estado de Tabasco. (2017). Ley de Protección Ambiental del Estado de Tabasco (2017). Periódico Oficial del Estado. México.

Centro de Estudios de las Finanzas Públicas (2016). La política ambiental y de combate al cambio climático en el Presupuesto de Egresos de la Federación. Extraído de: http://www.cefp.gob.mx/transp/CEFP-70-41-C-Estudio0018240718.pdf.Consulta: 24/06/18.

Comisión Nacional para el Conocimiento y Uso de la Biodiversidad. (2009). Capital natural en México. Síntesis: conocimiento actual, evaluación y perspectiva de sustentabilidad. Extraído de:: https://www.biodiversidad.gob.mx/pais/pdf/CapNatMex/Capital\%20Natural\% 20de\%20Mexico_Sintesis.pdf Consulta: 04/05/18.

Delgadillo, Luis. (2005). Principios de Derecho Tributario (Quinta ed.). Limusa. México.

Greenpeace (2016). La destrucción de México La realidad ambiental del país y el cambio climático. Extraído de: https://www.greenpeace.org/archivemexico/Global/mexico/report/2009/6/destruccion_mexico.pdf_Consulta: 04/06/18.

Hernández, Roberto, Fernández, Carlos y Baptista, Pilar (2016). Metodología de la investigación. McGraw Hill. México.

Instituto Nacional de Estadística y Geografía [INEGI] (2018). Resultados del Censo Nacional de Gobiernos Municipales y Delegacionales 2017. Extraído de: http://www.beta.inegi.org.mx/contenidos/saladeprensa/boletines/2018/EstSegP ub/cngmd2018 02.pdf. Consulta: 06/05/18.

Jofra, Marta y Puig-Ventosa, Ignasi. (2014). Fiscalidad ambiental e instrumentos de financiación de la economía verde. Fundación Fórum Ambiental. Extraído de: http://wp.forumambiental.org/wpcontent/uploads/2017/11/fiscalidad_ambiental.pdf Consulta: 06/06/18.

Labandeira, Xavier, León, Carmelo, y Vázquez, María. (2007). Economía Ambiental. Pearson Education. México.

Miller, Tyler. (2007). Ciencia ambiental: Desarrollo sostenible, un enfoque integral. Editores internacionales Thomson. México.

Organización de las Naciones Unidas. (1992). Convención marco de las naciones unidas. Extraído de: https://unfccc.int/resource/docs/convkp/convsp.pdf Consulta: 25/05/18.

Organización para la Cooperación y el Desarrollo Económico. (2001). Environmetally related taxes in the OECD countries. Extraído de: 
Impuestos ambientales sobre los desechos sólidos en tabasco: en pro del medio ambiente

http://www.oecd.org/environment/tools-evaluation/36966499.pdf. Consulta: $11 / 06 / 18$.

Pitrone, Federica. (2014). Definiendo los impuestos ambientales: Aportes desde el tribunal de justicia de la Unión Europea. IUS ET VERITAS. Número 49. Perú, (Pp.78-90).

Rosas, Aniceto y Santillán, Roberto. (1962). Teoría General de las Finanzas Públicas y el Caso de México. Escuela Nacional de Economía. México.

Ruiz, César. (2016). Derecho Tributario y Derechos Humanos. Tirant lo Blanch. México.

Santiago, Armando. (2009). La globalización del deterioro ambiental. Aldea mundo. 14(21). Venezuela. (Pp. 63-72).

Soto, Apolinar. (2007). Finanzas Públicas y las Funciones del Estado. Investigación y Ciencia. Número 37. México. (Pp.45-48).

Varela, Laura y García, María. (2010). La reforma fiscal verde en España: Un nuevo impulso fiscal: Boletín Económico de ICE 1(3002). Extraído de: http://www.revistasice.com/index.php/BICE/article/view/5011 Consulta: 07/05/18. 